\title{
Indicadores de calidad en la atención de salud en cirugía oncológica de cabeza y cuello
}

\section{Quality indicators in health care in head and neck cancer surgery}

\author{
Sebastián Castro M. ${ }^{1}$, Felipe Cardemil M. ${ }^{1,2,3}$
}

\section{Resumen}

La mayoría de los servicios de salud han experimentado un aumento de los costos asociados a la atención de salud lo que ha llevado a adoptar medidas para optimizar la costo-efectividad de los servicios otorgados. Desde esa perspectiva surge la atención de salud basada en el valor. El concepto de "calidad en la atención de salud" se ha definido como el grado en el cual los servicios de salud aumentan la posibilidad de generar ciertos desenlaces en salud a los que se aspira. Los indicadores de calidad de clasifican en indicadores de estructura, de proceso, y de desenlace. Los indicadores de estructura se refieren a las características del sistema de salud o de la institución hospitalaria. Los indicadores de proceso se refieren a los que el proveedor de servicios de salud realiza para el proceso de atención en salud, mientras que los indicadores de desenlace se refieren a los resultados del proceso en el paciente. El objetivo de la presente revisión es proveer un marco conceptual para dar un contexto al concepto de indicadores de calidad en salud y el rol que estos juegan en cirugía oncológica de cabeza y cuello. Se debe aspirar a lograr un mayor cumplimiento de los indicadores de calidad en cirugía oncológica de cabeza y cuello, especialmente en instituciones terciarias de referencia. Aplicar indicadores de calidad en el manejo oncológico en cabeza y cuello permitiría mejorar tanto la percepción y satisfacción del usuario, como también mejorar resultados oncológicos en estos pacientes.

Palabras clave: Cirugía oncológica de cabeza y cuello, indicadores de calidad, atención de salud basada en el valor.

\begin{abstract}
Most health services have experienced an increase in the costs associated with health care, which has led to the adoption of measures to optimize the cost-effectiveness of the services provided. From this perspective, the concept of value-based health care emerged. The concept of "quality in health care" has been defined as the degree to which health services increase the possibility of generating certain desired health outcomes. Quality indicators are classified into structure, process, and outcome indicators. The structure indicators refer to the characteristics of the health system or the hospital institution. Process indicators refer to those that the health service provider performs for the health care process, while outcome indicators refer to the results of the process in the patient. The objective of this review is to provide a conceptual framework to give a context to the concept of health quality indicators and the role they play in head and neck surgical oncology. The system should aspire to achieve greater compliance with quality indicators in head and neck cancer surgery, especially in referral tertiary institutions. Applying quality indicators in head and neck cancer management would improve both user perception and satisfaction, as well as improve oncological results in these patients.
\end{abstract}

Keywords: Head and neck surgical oncology, quality indicators, value-based health care.

\author{
'Departamento de \\ Otorrinolaringología, Facultad \\ de Medicina, Universidad de \\ Chile. Santiago, Chile. \\ ${ }^{2}$ Departamento de Oncología \\ Básico Clínica, Facultad de \\ Medicina, Universidad de \\ Chile. Santiago, Chile. \\ ${ }^{3}$ Departamento de \\ Otorrinolaringología, Clínica \\ Las Condes. Santiago, Chile. \\ Los autores declaran no tener \\ conflictos de interés.
}

Recibido el 13 de noviembre de 2020. Aceptado el 17 de enero de 2021

Correspondencia: Felipe Cardemil M. Departamento de Oncología Básico Clínica, Facultad de Medicina, Universidad de Chile. Santiago, Chile. Email: felipecardemil@med. uchile.cl 


\section{Introducción}

La mayoría de los servicios de salud del mundo occidental han experimentado un aumento de los costos asociados a la atención de salud en el último tiempo, lo que ha llevado a adoptar medidas para optimizar la costo-efectividad de los servicios otorgados. Desde esa perspectiva surge la atención de salud basada en el valor, definida como los resultados de la atención de salud por los recursos gastados para lograr esos resultados. Para lograr una mayor costo-efectividad del sistema es necesario evaluar tanto los resultados como los costos, con el fin de otorgar a la población los mejores resultados a los menores costos posibles. En ese contexto, surge la evaluación de la calidad de atención de salud ${ }^{1}$.

El concepto de "calidad en la atención de salud" se ha definido como el grado en el cual los servicios de salud para individuos y poblaciones aumentan la posibilidad de generar ciertos desenlaces en salud a los que se aspira, y que son consistentes con el estándar internacional esperado basado en el conocimiento profesional actual ${ }^{2}$. Una calidad inadecuada puede deberse a una sobre utilización, una subutilización, o una mal utilización de los servicios de salud.

Las razones del por qué es importante medir la calidad en la atención de salud son múltiples. Por un lado, puede ayudar a pacientes, instituciones pagadoras/aseguradoras, y proveedores de salud (individuales o institucionales), a tomar decisiones informadas respecto a un determinado tratamiento. Por otro lado, al medir la calidad se puede utilizar como parámetro de comparación para mejorar otros indicadores del proceso de atención de salud. Por último, es importante para determinar la utilidad de ciertos procesos en determinados flujogramas o programas de salud $^{2}$. Todo esto puede finalmente impactar en los resultados obtenidos en una atención de salud determinada.

Para medir la calidad en la atención de salud se deben establecer y utilizar medidas o métricas de calidad. Una medida de calidad es similar a un desenlace (outcome) en un ensayo clínico: es el cómo medimos el impacto de una determinada variable o intervención. En ese sentido, surge el concepto de indicadores de calidad, el cual se han extendido en varias áreas, incluido oncología de cabeza y cuello. En este artículo nos proponemos describir los indicadores de calidad en oncología de cabeza y cuello, evaluando su impacto real o potencial sobre los resultados oncológicos obtenidos frente a determinadas atenciones en salud.

\section{Indicadores de calidad}

Para referirnos a indicadores de calidad en salud utilizaremos el paradigma clásico propuesto por Donabedian ${ }^{3}$, que los clasifica en indicadores de estructura, de proceso, y de desenlace. Los indicadores de estructura se refieren a las características del sistema de salud o de la institución hospitalaria. Los indicadores de proceso se refieren a los que el proveedor de servicios de salud (proveedor institucional o individual) realiza para el proceso de atención en salud, mientras que los indicadores de desenlace se refieren a los resultados del proceso en el paciente. Cada tipo de indicador tiene una finalidad diferente: por ejemplo, para estimar resultados oncológicos como supervivencia o recurrencia, los indicadores de desenlace son los más importantes, pero para evaluar calidad de la atención en salud los indicadores más relevantes son los de proceso. Extrapolar resultados de indicadores de desenlace para evaluar calidad solamente puede ser válido cuando el desenlace que se está midiendo es un resultado directo de un proceso de atención de salud, sin otros factores externos que lo alteren ${ }^{2}$. Existe amplio material publicado en relación a la teoría de los indicadores y mediciones en salud, pero acá nos referiremos a ejemplos concretos de indicadores en cáncer de cabeza y cuello.

\section{De estructura}

Los indicadores de estructura reflejan el entorno en el que se enmarca la atención de salud, siendo esto la base para entregar una buena atención. Actualmente, se reconocen dos grandes factores que determinan este tipo de indicadores: el personal y el equipamiento. Por lo mismo, es en este punto donde se encuentran, el número de profesionales de salud o de instituciones para una población determinada, además de la red interdisciplinaria en el cual estos se encuentran. Estos indicadores 
por sí solos no garantizan calidad, e incluso pueden no representar buenos indicadores de la atención entregada ${ }^{1}$.

En relación a la atención en cáncer de cabeza y cuello, un tema importante es la regionalización de la atención, considerando la relativa baja incidencia de esta condición comparada con otros cánceres. Actualmente existe evidencia creciente que muestra que centralizar la atención en instituciones de mayor volumen mejoran los resultados oncológicos, disminuyendo el número de complicaciones asociadas a la atención de salud ${ }^{4}$. Por ejemplo, se ha descrito que la supervivencia en casos de cáncer laríngeo tanto avanzado como inicial es menor cuando se realiza en centros que no tienen un volumen de casos adecuado, o cuando se realiza en centros no terciarios ni académicos, así como se ha descrito que la supervivencia en casos de cáncer oral es mayor en centros que concentran más volumen de cirugías por año ${ }^{5}$. Además, se ha reportado que es más posible que el tratamiento para pacientes con cáncer de cabeza y cuello se ajuste a las guías y protocolos actuales cuando se lleva a cabo en centros de salud de referencia ${ }^{6}$. Estos centros deberían estar diseñados para albergar un equipo interdisciplinario que permita manejar los diferentes desafíos, inquietudes y problemas que presentan dichos pacientes, para que, de esa forma, este equipo logre una mayor experiencia y mejore la atención de salud que ofrece a los pacientes. Un ejemplo concreto de lo anterior es lo que ocurre en la provincia de Ontario, Canadá, que desde 1998 puso en marcha el Cancer Care Ontario (CCO), el cual ha permitido centralizar la atención del cáncer de cabeza y cuello en instituciones de referencia, mejorando sustantivamente los resultados oncológicos en esta población en las últimas décadas ${ }^{7}$.

\section{De proceso}

Los indicadores de proceso fueron hechos para evaluar la entrega de la atención de salud a la población. Estos incluyen todos los pasos de la trayectoria del manejo del paciente desde que ingresa al proceso en la institución o con el profesional, desde la apreciación de la lesión primaria tumoral, pasando por el procedimiento diagnóstico, tratamiento propuesto, seguimiento a largo plazo, y manejo de las complicaciones a largo plazo y supervivientes ${ }^{1}$. Es por esta razón que los mejores indicadores de proceso vienen de evidencia o estudios científicos en los cuales una determinada práctica resulta en un resultado favorable, con el agregado que debe existir idealmente consenso amplio en que esa determinada práctica es efectiva, reproducible y medible. Esto es parte de lo que se conoce como estándar de cuidado o de atención (standard of care). En ese sentido existen distintos puntos que es importante analizar en detalle.

\section{- Comité oncológico}

Existe evidencia creciente sobre el impacto de los equipos interdisciplinarios en la toma de decisiones en casos complejos, como ocurre en pacientes con cáncer de cabeza y cuello. En este sentido, uno de los aspectos relevantes es la presencia de comités oncológicos de cabeza y cuello (COCC) multidisciplinarios. Este comité debiese estar conformado por cirujanos oncólogos de cabeza y cuello (que debieran tener entrenamiento formal en oncología, ya sea desde la otorrinolaringología, la cirugía general, o la cirugía maxilofacial en países donde el entrenamiento es médico o con doble grado médico y dental), médicos oncólogos, radioterapeutas, radiólogos, y anatomopatólogos, todos idealmente especializados en cabeza y cuello, además de especialistas en cuidados paliativos, fonoaudiólogos, nutricionistas, patólogos orales, kinesiólogos, y enfermeros especializados en cabeza y cuello, junto con informáticos, epidemiólogos y bioestadísticos, los cuales analicen los datos que provengan del manejo clínico de estos pacientes. Es necesario que existan sesiones periódicas, habitualmente de forma semanal, con el fin de cubrir los casos nuevos que surjan en el centro en cuestión ${ }^{8}$.

El impacto positivo de los comités oncológicos sobre la toma de decisiones en pacientes con cáncer de cabeza y cuello se ha respaldado en la literatura. Por ejemplo, se ha visto que la presencia de los COCC puede variar tanto la etapificación como el manejo de los pacientes en hasta un $60 \%{ }^{9}$. Además, se ha visto que la presencia de los COCC puede tener un impacto en los desenlaces oncológicos finales, como quedó manifestado en el estudio retrospectivo de Liu y colaboradores publicado el 2019 donde se analizaban dos periodos en 
un mismo centro: previo a la presencia del COCC (pre-Comité) y posterior a la presencia del COCC (post-Comité). Este estudio incluyó 224 pacientes, 98 pacientes en la cohorte pre-Comité y 126 pacientes en la cohorte post-Comité. El promedio de seguimiento fue de 2,8 años y la mayoría de los casos fueron etapa avanzada (68\%). En el análisis KaplanMeier, la supervivencia general y específica de enfermedades mejoró significativamente en la cohorte post-Comité comparado con la cohorte pre-Comité, con una supervivencia específica de enfermedad a 5 años de un 75\% vs. $52 \%$, respectivamente, lo que refleja un menor riesgo de muerte de forma significativa, con un hazard ratio (HR) de 0,4810 .

No existe certeza de la razón por la cual el COCC se asociaría a una mejora en los desenlaces oncológicos finales. Se asume que se debe a una mayor exactitud tanto en el diagnóstico como en la etapificación, lo que llevaría a un refinamiento en los planes de manejo, logrando una mejora en los resultados finales. Además, el presentar un equipo interdisciplinario permitiría una mejor coordinación entre diferentes servicios, lo que llevaría a un menor tiempo para ejecutar tratamientos adyuvantes complementarios. Es por ello, que actualmente se considera como indicador de calidad el tener un comité oncológico a cargo de las decisiones terapéuticas en casos de pacientes con cáncer de cabeza y cuello ${ }^{11,12}$.

\section{- Adherencia a guías de práctica clínica}

Las guías de práctica clínica (GPC) conforman la base para estandarizar y optimizar la entrega de una atención en salud. Estas son desarrolladas en base a la evidencia, utilizando la mejor información disponible hasta la fecha de publicación. Por este motivo se consideran un indicador de proceso. Sin embargo, su aplicación debe tomarse con cuidado, considerando que las poblaciones objetivo pueden ser diferentes a las poblaciones de los propios estudios que justifican las recomendaciones de las GPC, por lo que cada caso debe ser analizado de manera individual ${ }^{1}$.

La adherencia a las recomendaciones de las GPC disminuiría la variabilidad de tratamiento y mejoraría el enfrentamiento basado en la evidencia ${ }^{7}$. Gourin y colaboradores publicaron un estudio retrospectivo el año 2015 donde analizaban la asociación entre la adherencia a guías clínicas nacionales norteamericanas, específicamente a la National Comprehensive Cancer Network (NCCN) y resultados a corto y largo plazo, además de los costos asociados, en pacientes con diagnóstico de carcinoma escamoso laríngeo entre los años 2004 y 2007, logrando un tamaño muestral de 2.370 pacientes. Ellos demostraron que un tratamiento de alta calidad (con mayor adherencia a las guías clínicas) se asociaba con menor probabilidad de pérdida de peso (odds ratio [OR] 0,6 [0,5$0,8]$ ), estenosis esofágica ( OR $0,5[0,3-0,8])$, dependencia a gastrostomía (OR $0,5[0,4-0,7]$ ), obstrucción de vía aérea (OR 0,7 [0,6-0,9]), traqueostomía (OR 0,5 [0,3-0,7]), y neumonía (OR 0,7 [0,5-0,9]). Además, demostraron una asociación entre una atención de alta calidad con un menor riesgo de muerte frente a pacientes que presentaban disfagia (hazard ratio [HR] 0,7 [0,6-0,8]), pérdida de peso (HR 0,8 $[0,6-0,9])$, obstrucción de vía aérea (HR 0,7 $[0,6-0,8])$, traqueostomía (HR 0,7 [0,5-0,9]), y neumonía (HR 0,8 $[0,6-0,9])^{13}$. Lo interesante de este estudio es que corresponde a la única publicación a la fecha en relación a cáncer de cabeza y cuello en conocimiento de los autores de la presente revisión, que relaciona la adherencia a guías clínicas con un mejor resultado clínico.

\section{- Tiempo de inicio del tratamiento inicial y adyuvante}

Un punto importante es el tiempo de inicio de tratamiento desde el diagnóstico inicial, debido a que, en oncología de cabeza y cuello, así como en otros tipos de cáncer, el manejo de los tiempos es fundamental. Lo anterior debido a la naturaleza rápidamente progresiva de esta enfermedad en un área anatómica compleja y funcionalmente vulnerable, lo que tiene un impacto directo en la supervivencia y en la calidad de vida ${ }^{1}$.

Una de las formas para abordar esta situación es mejorando la derivación desde la atención primaria de salud, disminuyendo los tiempos de espera. Es así como, por ejemplo, la UK National Institute for Health and Care Excellence (NICE) en 2005, publicó la primera guía de práctica clínica para el reconocimiento y derivación de un cáncer sospechoso, la cual sufrió una actualización el año 2015 por parte 
del departamento de salud, especificando tiempos en el cual el paciente con sospecha de cáncer debe ser visto, donde el objetivo nacional es de 14 días desde la derivación por parte de atención primaria. A pesar de que estos tiempos de espera bien establecidos, su verdadero impacto aún no es bien conocido ${ }^{14}$.

En cuanto al tiempo de espera desde el diagnóstico al inicio del tratamiento, este no ha sido establecido propiamente tal con un nivel de evidencia I, sin embargo, existen estudios que intentan definir un umbral demostrando su impacto en la supervivencia. Murphy y colaboradores publicaron el 2016 un estudio retrospectivo utilizando la National Cancer Data Base (NCDB) en pacientes con carcinoma escamoso de cabeza y cuello que recibieron tratamiento con intención curativa. Se analizaron 51.655 pacientes, de los cuales se determinó que un tiempo de inicio de tratamiento de 61 a 90 días versus menos de 30 días incrementaba el riesgo de mortalidad de forma independiente (HR 1,13). Además, determinaron rangos de umbrales, entre 46 a 52 días y entre 62 y 67 días, siendo más consistente el incremento del riesgo de muerte más allá de los 60 días ${ }^{15}$.

Otros tiempos relevantes son el tiempo entre la cirugía y el inicio de la radioterapia adyuvante cuando está indicada, y el tiempo entre la cirugía hasta completar la terapia adyuvante cuando esta sea necesaria, este último también llamado como tiempo de empaque (package time $)^{16}$. A pesar de haber cierta heterogeneidad en la definición, es mayoritariamente aceptado que el tiempo entre la cirugía y la terapia adyuvante debe estar circunscrito a un plazo menor o igual a seis semanas, lo que ha demostrado tener un impacto en reducir el riesgo de muerte en pacientes tratados quirúrgicamente por carcinoma escamoso de cabeza y cuello ${ }^{16}$. Por otro lado, con respecto al tiempo de empaque, este no está bien establecido teniendo una definición variable entre 77 a 100 o más días $^{16}$. A pesar de que su impacto no está bien establecido, en estudios observacionales se ha visto una menor supervivencia en aquellos pacientes con un tiempo de espera hasta completar el tratamiento mayor a 12 semanas ${ }^{17}$.

\section{- Cirugía oncológica y criterios de calidad}

En cuanto a los criterios de calidad quirúrgicos, es importante señalar que actualmente no existen criterios de calidad adoptados por alguna institución nacional de calidad para evaluar este ámbito. Sin embargo, se han intentado determinar posibles indicadores qué tendrían un impacto concreto sobre los resultados oncológicos.

En ese sentido, Cramer y colaboradores, publicaron el 2017 un estudio donde analizaron el impacto sobre los resultados oncológicos de cinco indicadores de calidad en pacientes con carcinoma escamoso de cabeza y cuello que fueron sometidos a tratamiento quirúrgico, específicamente sobre la supervivencia general, utilizando la base de datos de la NCDB entre 2004 y 2014 . Estos cinco indicadores fueron: márgenes quirúrgicos negativos; disección cervical con un número mayor o igual a 18 linfonodos; acceso a radioterapia adyuvante si estaba indicada; acceso a quimiorradioterapia adyuvante si estaba indicada; y comienzo de la terapia adyuvante dentro de un período de 6 semanas posterior a la cirugía. Se consideró como manejo de alta calidad si es que este cumplía más de $75 \%$ de los indicadores. Se incluyeron 76.853 pacientes en este estudio, donde se observó que el cumplimiento de estos factores en forma individual redujo el riesgo de muerte de forma significativa. Además, se determinó que los centros académicos y/o de alto volumen, definido como centro con más de 160 cirugías en un periodo de 10 años, presentaban mejores resultados oncológicos comparados con centros no académicos y de bajo volumen. Al analizar a aquellos que tuvieron un manejo de alta calidad el riesgo ajustado de muerte se redujo en un 19\%, siendo más probable recibir un manejo de alta calidad en centros de mayor volumen. Por su impacto sobre los resultados oncológicos, estos indicadores son susceptibles de ser adoptados para su utilización en la evaluación de calidad, sin embargo, están limitados al no considerar pacientes que se sometieron a radioterapia o quimiorradioterapia primaria ${ }^{18}$. Sin embargo, es interesante destacar que este tipo de métricas es fácilmente medible y podrían ser evaluadas a nivel nacional o regional para ser consideradas como criterios de calidad en cirugía oncológica.

Por otro lado, también se ha intentado determinar indicadores de calidad en el caso de la reconstrucción con colgajos microvas- 
culares libres en cirugía de cabeza y cuello. Eskander y colaboradores publicaron el 2018 un estudio retrospectivo donde analizaban 515 pacientes que se sometieron a reconstrucción en cabeza y cuello mediante colgajos libres en la Ohio State University entre el 2006 y 2012, con el fin de determinar predictores de mayor estadía hospitalaria, readmisión dentro de 30 días e ingreso a pabellón dentro de 30 días. Ellos determinaron que $31,5 \%$ de los pacientes tuvieron una estadía hospitalaria mayor a 9 días, donde los predictores fueron tener una localización tumoral en cavidad oral y faringe, transfusión sanguínea, diabetes mellitus o tener cualquier tipo de complicación (tanto médica como quirúrgica). Un $12,6 \%$ de los pacientes fueron readmitidos dentro de 30 días, donde los predictores fueron ausencia de evaluación preoperatoria y el haber presentado cualquier tipo de complicación posoperatoria. Finalmente, $14,8 \%$ de los pacientes tuvieron que ingresar nuevamente a pabellón, donde el predictor fue la edad avanzada. Por esta razón los autores señalan que un indicador de calidad debería ser la evaluación clínica preoperatoria, por su impacto sobre la readmisión temprana. Por otro lado, señalan que se deberían hacer esfuerzos para de encontrar predictores precoces de complicación, debido a que este fue uno de los factores pronósticos más importantes en este estudio ${ }^{19}$.

\section{De resultado}

El último grupo de indicadores corresponde a los indicadores de resultado o desenlace, los que se refieren a la evaluación del resultado final de la atención de salud. Es por esta razón que históricamente el indicador de mayor importancia en pacientes oncológicos ha sido la supervivencia. En este punto es importante señalar que a pesar de que en el último tiempo se ha mejorado la tecnología, especialmente en relación al tratamiento con radioterapia o quimiorradioterapia, esta no sólo se enfoca en mejorar la supervivencia propiamente tal, sino además en disminuir la toxicidad tanto aguda como tardía de dicha intervención ${ }^{1}$. Es por esto que actualmente se está tomando en cuenta otro aspecto relevante en este tipo de pacientes, que es la calidad de vida, la salud mental en los supervivientes y los aspectos psico-oncológicos $\mathrm{y}$ los resultados funcionales posteriores al tra- tamiento oncológico ofrecido. La evaluación de este punto se realiza mediante encuestas de calidad de vida, las cuales tienen presentan algunos desafíos en su implementación, como poca adherencia a contestarlas entre otras ${ }^{1}$.

Otro punto importante para los indicadores de desenlace es la evaluación de los eventos adversos a los tratamientos oncológicos en oncología de cabeza y cuello. Esto es de gran importancia al considerar que los tratamientos relacionados con los eventos adversos corresponden a la $14^{\text {ta }}$ causa más común de carga de enfermedad a nivel mundial ${ }^{20}$. En el caso particular de cáncer de cabeza y cuello estos eventos adversos son bien conocidos, tanto relacionados con el tratamiento quirúrgico como frente a quimioterapia o radioterapia, los cuales deben ser reportados y registrados de forma sistemática, con el fin de determinar déficits y posibles estrategias para mejorar los resultados de un servicio en particular. Dentro de los efectos adversos se encuentran derivados de la cirugía resectiva, como dificultades funcionales, cicatrización, alteraciones cosméticas y de comunicación, así como otros derivados de la radio terapia o la quimioterapia como fibrosis, osteorradionecrosis y xerostomía, entre otros. El concepto que engloba la evaluación de la calidad de vida y de los aspectos funcionales, así como de la salud mental y el manejo de las complicaciones son los policlínicos de "survivorship", donde los pacientes son evaluados periódicamente en paralelo a los controles oncológicos para evaluar y manejar los aspectos recientemente mencionados.

\section{Importancia de los indicadores de calidad}

Los indicadores antes nombrados presentan un impacto oncológico concreto en el manejo y los resultados de los pacientes con cáncer de cabeza y cuello, tanto en supervivencia general o específica de enfermedad, como en la calidad de vida del paciente, lo que corresponde a la razón principal que justifica el uso de indicadores de calidad. Esto permite además poder estandarizar el análisis de los resultados y realizar comparaciones más válidas. Esto ha sido demostrado en otras áreas quirúrgicas, donde se ha visto que la adop- 
ción de indicadores de calidad en diferentes centros mejora los resultados quirúrgicos, disminuyendo el número de complicaciones, reduciendo además los costos asociados a la atención de salud ${ }^{21}$. Por este motivo, en el año 2007 la American Head and Neck Society aprobó el primer grupo de indicadores de calidad para cáncer oral, y posteriormente para cáncer laríngeo el año 2009. Ambos grupos de indicadores son muy similares e incluyen aspectos como documentación de anatomía patológica, etapa diagnóstica, consejería para cesación de tabaco, evaluación interdisciplinaria por cirugía oncológica, oncología médica y radio oncología, y seguimiento estandarizado para detección temprana de recurrencias, segundos primarios, complicaciones e hipotiroidismo en caso de radioterapia (Tablas 1 y 2 ) $^{6}$.

Derivado de esas recomendaciones, el estudio mencionado previamente realizado por Cramer utilizó como base estas recomendaciones para evaluar los cinco indicadores de calidad en carcinoma escamoso de cabeza y cuello tratado quirúrgicamente. Este estudio evaluó la adherencia a dichos indicadores estimado que era de un $80 \%$ para márgenes quirúrgicos negativos, $73,1 \%$ para disección cervical eficiente (18 linfonodos disecados o más), 69\% para radioterapia adyuvante, $42,6 \%$ para quimioterapia adyuvante, y $44,5 \%$ para terapia adyuvante dentro de 6 semanas, este último con una tendencia a la baja en la última década ${ }^{18}$.

De forma más reciente, el grupo de trabajo del proyecto RARECAREnet, compuesto por Trama y colaboradores, han elaborado a través de un panel de expertos multidisciplinarios en el manejo de cáncer de cabeza y cuello, once indicadores de calidad que abordan el cuidado de pacientes con cáncer de cabeza y cuello en relación tanto al diagnóstico como al tratamiento, con un enfoque en cirugía y radioterapia, no abordando quimioterapia $(\text { Tabla } 3)^{11}$.

Tabla 1. Medidas de calidad de la American Head and Neck Society para carcinoma escamoso de cavidad oral

\section{Medidas previas al tratamiento de la cavidad oral:}

1. Todos los pacientes con cáncer de lengua oral requieren documentación de patología utilizando los criterios del College of American Pathologists (CAP) con confirmación histopatológica de la enfermedad

2. Todos los pacientes con cáncer de lengua oral requieren documentación de la estadificación TNM adecuada (según lo define el American Joint Committee on Cancer)
a. Evaluación del tamaño del tumor primario $(T)$
b. Evaluación de los grupos ganglionares regionales para la linfadenopatía metastásica (N)
c. Evaluación de enfermedad sistémica (M)

3. Consejería para dejar de fumar

\section{Medidas de calidad relacionadas con el tratamiento:}

1. Todos los pacientes con cáncer de cavidad oral con estadio T avanzado o ganglios linfáticos metastásicos deben ser derivados a oncología radioterápica para considerar la radioterapia posoperatorio

2. Todos los pacientes con cáncer de cavidad oral con márgenes patológicos positivos o ganglios linfáticos metastásicos que muestren extensión extracapsular deben ser derivados a un oncólogo médico u oncólogo radioterapeuta para que los considere para quimioterapia y radiación adyuvantes

\section{Medidas de calidad posteriores al tratamiento:}

1. Todos los pacientes tratados por cáncer de cavidad oral deben tener visitas de seguimiento para el manejo de los síntomas y la vigilancia de la recurrencia y segundos tumores primarios

2. Los pacientes tratados con radioterapia dirigida al cuello deben someterse a una evaluación de la hormona estimulante del tiroides (TSH) sérica para detectar hipotiroidismo. Se debe controlar la TSH sérica posterior al tratamiento dentro de los doce meses posteriores a la finalización de la radioterapia

Adaptado de Chen AY. The development of quality of care measures for oral cavity cancer. Arch Otolaryngol Head Neck Surg. 2008;134(6):6726 
Tabla 2. Medidas de calidad de la American Head and Neck Society para carcinoma escamoso de laringe

\section{Medidas previas al tratamiento del cáncer de laringe:}

1. Todos los pacientes con cáncer de laringe requieren documentación de la patología utilizando los criterios CAP con confirmación histopatológica de la enfermedad

2. Todos los pacientes con cáncer de laringe requieren documentación de la estadificación TNM apropiada (según lo define el American Joint Committee on Cancer)

a. Evaluación del tamaño del tumor primario $(\mathrm{T})$

b. Evaluación de los grupos ganglionares regionales para la linfadenopatía metastásica (N)

c. Evaluación de enfermedad sistémica (M)

3. Asesoramiento para dejar de fumar

4. Asesoramiento previo a la laringectomía para pacientes sometidos a laringectomía.

\section{Medidas de calidad relacionadas con el tratamiento:}

1. Todos los pacientes con cáncer de laringe en estadio $T$ avanzado deben ser derivados a oncología radioterápica para considerar la radioterapia posoperatoria

2. Todos los pacientes con cáncer de laringe posoperatorio con más de un ganglio linfático positivo y / o estadio T avanzado deben remitirse a oncología radioterápica para considerar la radioterapia

3. Todos los pacientes con cáncer de laringe con márgenes patológicos positivos o ganglios linfáticos metastásicos que muestren extensión extracapsular deben ser derivados a un oncólogo médico o un oncólogo radioterapeuta para que los consideren para quimioterapia y radiación adyuvantes

4. Todos los pacientes que se someten a una cirugía de laringe (parcial o total) deben ser evaluados y seguidos por un fonoaudiólogo

\section{Medidas de calidad posteriores al tratamiento:}

1. Todos los pacientes tratados por cáncer de cavidad oral deben tener visitas de seguimiento para el manejo de los síntomas y la vigilancia de la recurrencia y segundos tumores primarios

2. Los pacientes tratados con radioterapia dirigida al cuello deben someterse a una evaluación de la TSH sérica para detectar hipotiroidismo. Se debe verificar la TSH sérica posterior al tratamiento dentro de los 12 meses posteriores a la finalización de la radioterapia

Adaptado de Cramer JD, Speedy SE, Ferris RL, Rademaker AW, Patel UA, Samant S. National evaluation of multidisciplinary quality metrics for head and neck cancer. Cancer. 2017;123(22):4372-4381'18.

Este mismo grupo de trabajo realizó un estudio observacional incorporando los registros de cáncer de cuatro países europeos (Italia, Holanda, Eslovenia y Escocia) para evaluar el grado de cumplimiento por país de cada indicador de calidad, específicamente para carcinoma escamoso de laringe, cavidad oral, orofaringe e hipofaringe. En cuanto a sus resultados, en relación al diagnóstico, se observó que en la mayoría de los países sus pacientes fueron etapificados antes del tratamiento, sin embargo, se detectó que en Escocia la proporción de pacientes sin etapificación previa fue de un $22 \%$ para laringe y $24 \%$ para otros sitios. Por otro lado, en relación con el manejo, se observó que un gran porcentaje de pacientes comenzaron a recibir su tratamiento con intención curativa después de cuatro semanas del diagnóstico; por ejemplo, Italia tuvo un $60 \%$ de pacientes que empezaron dentro del período del mes, mientras que en los otros países solo un $40 \%$ comenzó su tratamiento de forma precoz. En cuanto a la decisión acerca del tipo de manejo, se observó que la adherencia a guías de práctica clínica fue alta en pacientes con enfermedad localizada con un rango entre $72 \%-79 \%$; sin embargo, fue baja en enfermedad avanzada con un rango entre un $19 \%-44 \%{ }^{11}$. En relación a la cirugía, sólo un 56\% en Escocia y un 70\% en Holanda y Eslovenia obtuvieron márgenes negativos en casos de cirugía con intento curativo. En 


\section{Tabla 3. Indicadores de calidad para cáncer de cabeza y cuello}

Diagnóstico

1. Porcentaje de pacientes con una etapa definida en el momento del diagnóstico.

Tiempo para comenzar el tratamiento y el cumplimiento del tratamiento con las guías clínicas

2. Tiempo para comenzar el tratamiento (tiempo entre el diagnóstico patológico definitivo y el comienzo de la cirugía o radioterapia $<1$ mes)

3. Tiempo de inicio de la radioterapia posoperatoria o la quimioterapia concomitante (< 8 semanas desde la cirugía)

4. Porcentaje de pacientes con estadios tempranos I y II referidos para cirugía o radioterapia

5. Porcentaje de pacientes con estadio III y IV localmente avanzado remitidos para cirugía más radioterapia posoperatoria o quimiorradioterapia posoperatoria o quimiorradioterapia concomitante. Calidad de la cirugía y radioterapia

6. Porcentaje de resección tumoral completa (verificación histológica de márgenes libres de tumor después de la cirugía)

7. Porcentaje de reoperación dentro de los 30 días posteriores a la cirugía principal

8. Porcentaje de toxicidades tardías de grado $\geq 3$ ( $>3$ meses después de la radioterapia)

9. Porcentaje de pacientes que reciben radioterapia de intensidad modulada frente al \% que recibe radioterapia conformada 3D

10. Porcentaje de pacientes que reciben la cirugía apropiada para su etapa (p. Ej., cirugía mínimamente invasiva y reconstructiva). Calidad de los informes de patología después de la cirugía

11. Porcentaje de informes de patología después de la cirugía con un conjunto completo de elementos de datos básicos registrados. Según el Royal College of Pathologists: sitio y lateralidad del carcinoma, diámetro máximo del tumor, profundidad máxima de la invasión, tipo histológico de carcinoma, grado de diferenciación (grado), patrón de invasión, estado del margen, afectación ganglionar

Disponibilidad de decisión multidisciplinaria formalizada (con miembros expertos en cánceres de cabeza y cuello)

Participación en investigación clínica y traslacional

Modificado y traducido de publicación de Trama A, Botta L, Foschi R, et al. Quality of Care Indicators for Head and Neck Cancers: The Experience of the European Project RARECAREnet. Front Oncol. 2019;9:83711.

relación a la radioterapia, la mayoría de los pacientes empezaban radioterapia adyuvante posterior a cirugía antes de 8 semanas, variando desde un $52 \%$ en Italia y $79 \%$ en Holanda. No obstante, un aspecto interesante fue que contraviniendo las recomendaciones del Royal College of Pathologists, sólo una minoría de los informes de la biopsia operatoria incluía toda la información histopatológica necesaria para tomar una decisión terapéutica ( $1 \%$ en Eslovenia a $24 \%$ en Italia $)^{11}$.

Por lo tanto, existen varios aspectos por mejorar en cuanto el cuidado de los pacientes con cáncer de cabeza y cuello. Sin embargo, se debe aspirar a lograr un mayor cumplimiento de estos indicadores de calidad, especialmente en instituciones terciarias de referencia, que, con un mayor volumen de pacientes, junto con un equipo interdisciplinario organi- zado, lograrán mejores resultados para los pacientes.

\section{Conclusión}

El manejo de los pacientes con cáncer de cabeza y cuello es complejo, requiriendo el análisis de diferentes variables con el fin de tomar las mejores decisiones en cada caso en particular. Actualmente, es necesario tener estándares de calidad basados e informados por evidencia, que permitan abordar de forma más adecuada y eficiente al paciente y a su enfermedad. Es por esta razón que aplicar indicadores de calidad en el manejo de pacientes oncológicos en cabeza y cuello permitiría mejorar tanto la percepción y satisfacción del usuario, como también mejorar resultados oncológicos de estos pacientes. 


\section{Bibliografía}

1. Takes RP, Halmos GB, Ridge JA, et al. Value and Quality of Care in Head and Neck Oncology. Curr Oncol Rep. 2020;22(9):92. doi: 10.1007/s11912-02000952-5.

2. Chen AY. Quality initiatives in head and neck cancer. Curr Oncol Rep. 2010;12(2):109-114. doi: 10.1007/ s11912-010-0083-6.

3. Donabedian A. The Definition of Quality and Approaches in Assessment, Vol. 1. Ann Arbor, MI: Health Administration Press; 1980.

4. Chen AY, Halpern M. Factors predictive of survival in advanced laryngeal cancer. Arch Otolaryngol Head Neck Surg. 2007;133(12):1270-1276. doi: 10.1001/ archotol.133.12.1270.

5. Eskander A, Merdad M, Irish JC, et al. Volumeoutcome associations in head and neck cancer treatment: a systematic review and meta-analysis. Head Neck. 2014;36(12):1820-1834. doi: 10.1002/ hed.23498.

6. Chen AY. The development of quality of care measures for oral cavity cancer. Arch Otolaryngol Head Neck Surg. 2008;134(6):672. doi: 10.1001/ archotol.134.6.672.

7. Eskander A, Goldstein DP, Irish JC. Health Services Research and Regionalization of Care-From Policy to Practice: the Ontario Experience in Head and Neck Cancer. Curr Oncol Rep. 2016;18(3):19. doi: 10.1007/ s11912-016-0500-6.

8. James, Nick \& Hartley, Andrew. Improving Outcomes in Head and Neck Cancer - The Manual. Clinical oncology (Royal College of Radiologists (Great Britain)). 2003; 15. 264-5. doi: 10.1016/S09366555(03)00167-5.

9. Bergamini C, Locati L, Bossi P, et al. Does a multidisciplinary team approach in a tertiary referral centre impact on the initial management of head and neck cancer?. Oral Oncol. 2016;54:54-57. doi: 10.1016/j.oraloncology.2016.01.001.

10. Liu JC, Kaplon A, Blackman E, Miyamoto C, Savior $\mathrm{D}$, Ragin C. The impact of the multidisciplinary tumor board on head and neck cancer outcomes. Laryngoscope. 2020;130(4):946-950. doi: 10.1002/ lary.28066.

11. Trama A, Botta L, Foschi R, et al. Quality of Care Indicators for Head and Neck Cancers: The Experience of the European Project RARECAREnet.
Front Oncol. 2019;9:837. doi: 10.3389/

fonc.2019.00837.

12. van Overveld LF, Braspenning JC, Hermens RP. Quality indicators of integrated care for patients with head and neck cancer. Clin Otolaryngol. 2017;42(2):322-329. doi: 10.1111/coa.12724.

13. Gourin CG, Starmer HM, Herbert RJ, et al. Quality of care and short- and long-term outcomes of laryngeal cancer care in the elderly. Laryngoscope. 2015;125(10):2323-2329. doi: 10.1002/lary.2537.

14. Langton S, Siau D, Bankhead C. Two-week rule in head and neck cancer 2000-14: a systematic review. $\mathrm{Br}$ J Oral Maxillofac Surg. 2016;54:120-31.

15. Murphy CT, Galloway TJ, Handorf EA, et al. Survival Impact of Increasing Time to Treatment Initiation for Patients With Head and Neck Cancer in the United States. J Clin Oncol. 2016;34(2):169-178. doi: 10.1200/ JCO.2015.61.5906.

16. Graboyes EM, Kompelli AR, Neskey DM, et al. Association of Treatment Delays With Survival for Patients With Head and Neck Cancer: A Systematic Review. JAMA Otolaryngol Head Neck Surg. 2019;145(2):166-177. doi: 10.1001/ jamaoto.2018.2716.

17. Goel AN, Frangos MI, Raghavan G, et al. The impact of treatment package time on survival in surgically managed head and neck cancer in the United States. Oral Oncol. 2019;88:39-48. doi: 10.1016/j. oraloncology.2018.11.021.

18. Cramer JD, Speedy SE, Ferris RL, Rademaker AW, Patel UA, Samant S. National evaluation of multidisciplinary quality metrics for head and neck cancer. Cancer. 2017;123(22):4372-4381. doi: 10.1002/cncr.30902.

19. Eskander A, Kang SY, Tweel B, et al. Quality Indicators: Measurement and Predictors in Head and Neck Cancer Free Flap Patients. Otolaryngol Head Neck Surg. 2018;158(2):265-272. doi: 10.1177/0194599817742373.

20. Jha AK, Larizgoitia I, Audera-Lopez C, PrasopaPlaizier N, Waters H, Bates DW. The global burden of unsafe medical care: analytic modelling of observational studies. BMJ Qual Saf. 2013;22:809-15.

21. Hall BL, Hamilton BH, Richards K, Bilimoria KY, Cohen ME, Ko CY. Does surgical quality improve in the American College of Surgeons National Surgical Quality Improvement Program: an evaluation of all participating hospitals. Ann Surg. 2009;250(3):363376. doi: 10.1097/SLA.0b013e3181b4148f. 